\title{
Theoretical Study of High-Frequency Response of InGaAs/AlAs Double-Barrier Nanostructures
}

\author{
Konstantin S. Grishakov, ${ }^{1,2}$ Vladimir F. Elesin, ${ }^{1}$ \\ Mikhail M. Maslov, ${ }^{1,2}$ and Konstantin P. Katin ${ }^{1,2}$ \\ ${ }^{1}$ Department of Condensed Matter Physics, National Research Nuclear University MEPhI (Moscow Engineering Physics Institute), \\ Kashirskoe Sh. 31, Moscow 115409, Russia \\ ${ }^{2}$ Laboratory of Computational Design of Nanostructures, Nanodevices and Nanotechnologies, Research Institute for \\ the Development of Scientific and Educational Potential of Youth, Aviatorov Str. 14/55, Moscow 119620, Russia
}

Correspondence should be addressed to Mikhail M. Maslov; mike.maslov@gmail.com

Received 2 May 2017; Accepted 5 June 2017; Published 6 July 2017

Academic Editor: Francesco Ruffino

Copyright (C) 2017 Konstantin S. Grishakov et al. This is an open access article distributed under the Creative Commons Attribution License, which permits unrestricted use, distribution, and reproduction in any medium, provided the original work is properly cited.

\begin{abstract}
The presented article contains the numerical calculations of the InGaAs/AlAs resonant tunneling diode's (RTD) response to the AC electric field of a wide range of amplitudes and frequencies. These calculations have been performed within the coherent quantummechanical model that is based on the solution of the time-dependent Schrödinger equation with exact open boundary conditions. It is shown that as the field amplitude increases, at high frequencies, where $\hbar \omega>\Gamma$ ( $\Gamma$ is the width of the resonant energy level), the active current can reach high values comparable to the direct current value in resonance. This indicates the implementation of the quantum regime for RTD when radiative transitions are between quasi-energetic levels and the resonant energy level. Moreover, there is an excitement of higher quasi-energetic levels in AC electric fields, which in particular results in a slow droop of the active current as the field amplitude increases. It also results in potentially abrupt changes of the operating point position by the $\hbar \omega$ value. This makes it possible to achieve relatively high output powers of InGaAs/AlAs RTD having an order of $10^{5} \mathrm{~W} / \mathrm{cm}^{2}$ at high frequencies.
\end{abstract}

\section{Introduction}

The resonant tunneling diode is a two-barrier nanostructure. It operates on the principle of electron transport through resonant energy levels. These energy levels are formed in a quantum well due to the interference of electrons. Such electron transport leads to the appearance of the negative differential conductivity (NDC) area at the current-voltage characteristic ( $I-V$ curve) of RTD. This $I-V$ curve enables the generation of an electromagnetic field. The fact that the resonant tunneling diode has NDC area was experimentally demonstrated at liquid nitrogen temperatures $(77 \mathrm{~K})$ in [1] and the same at room temperature in [2].

A resonant tunneling diode is a compact solid electronic device operated at room temperature and is a possible candidate to be a source of terahertz radiation. The generation of electromagnetic waves within an RTD was obtained at about $200 \mathrm{~K}$ in 1984 [3], wherein the frequency and output power were low $18 \mathrm{GHz}$ and some microwatts, respectively. In recent years, through the reduction of the thickness of the quantum well and barriers [4], the optimisation of the collector spacer layer thickness [5], and the size of the slot antenna [6], a significant growth of frequency has been achieved. Currently, the oscillation frequency of GaAs-based RTD equal to $1.92 \mathrm{THz}$ has been achieved [7]. It is the highest one ever recorded for electronic devices operating at room temperature. The output power in the latter case was about $0.4 \mu \mathrm{W}$.

Despite the obvious recent progress in the frequency characteristics of InGaAs/AlAs resonant tunneling diodes, the output powers within a terahertz range are still low (some microwatts). Also, there is still a pending problem of RTD's high-frequency features. This results in the growing importance of theoretical studies aimed at researching the behaviour of a resonant tunneling diode in a high-frequency electromagnetic field. 
Since an RTD works based upon quantum effects, the most consistent model for the theoretical description thereof is the so-called coherent model based upon the solution of the time-dependent Schrödinger equation with open boundary conditions. For the first time, RTD was described using this model in $[8,9]$ that used numerical calculations. Furthermore, it is also necessary to note analytical work [10] that developed an effective method to research RTDs in strong electromagnetic fields.

An important result obtained within the coherent model is the assumption that there does exist a quantum regime for RTD [11] associated with photon emission through resonant transitions between quasi-energetic levels appearing in an AC electric field [12] and resonant energy level in a quantum well. In this case, active current $J_{c}$ reaches its maximum at the finite frequency when the condition $\varepsilon-\varepsilon_{R} \approx \hbar \omega>\Gamma$ is complied with, that is, beyond the area of the maximum NDC. Here, $\varepsilon$ is the energy of the emitter's electrons, $\varepsilon_{R}$ and $\Gamma$ are the energy and the width of the resonant level, respectively, and $\omega$ is the frequency of the AC electric field. However, in a "classical" regime (as defined in [11]), where the operating point position is selected in the maximum NDC, $J_{c}$ is at its highest within the low-frequency limits, whereas its value drops as the frequency increases as $1 / \omega^{4}$. Thus, at high frequencies, $\hbar \omega>$ $\Gamma$ active current in quantum regime is much higher than the active current in "classical" regime. Subsequently, the results of [11] obtained for a weak field having the field potential amplitude $V_{\text {ac }} \ll \Gamma$ were confirmed by numerical calculations of [13] and summarised in [14] for a wider range of field amplitudes limited by the condition $V_{\mathrm{ac}}<\varepsilon_{R}$. The latter condition is always complied with RTD. It resulted that, in quantum regime, the dependence of the active current on the amplitude of the AC electric field coincides with the intensity distribution at the Fraunhofer diffraction, which indicates the interference of electrons absorbing and emitting photons. Moreover, current $J_{c}$ reaches a high value approximately equal to half of the maximum direct current of the RTD. The results of $[11,13,14]$ were obtained in a simplified model with $\delta$-functional barriers and monoenergetic electrons (i.e., it was believed that the structure was bombarded by a flow of electrons having the assigned density with a fixed value of the energy) and in the absence of the DC voltage.

Subsequently, $[15,16]$ using computer simulation studied the behaviour of InGaAs/AlAs RTD in an AC electric field in the model that more accurately coincides with the experiment with square barriers of the finite width and height, with the Fermi distribution of electrons over the energy states as well as in the presence of the DC voltage. They used a numerical solution of the time-dependent Schrödinger equation based upon the expansion of the wave function under the Floquet modes. However, in these studies, much attention is paid to double-well structures of RTDs and the opportunities to detect the alternating current signal using these double-well structures.

The purpose of this work is to calculate, using computer simulation, the InGaAs/AlAs RTD response to an AC electric field within a wide range of frequency and field amplitudes, taking into consideration the Fermi distribution of electrons over the energy states and in the presence of the DC voltage.

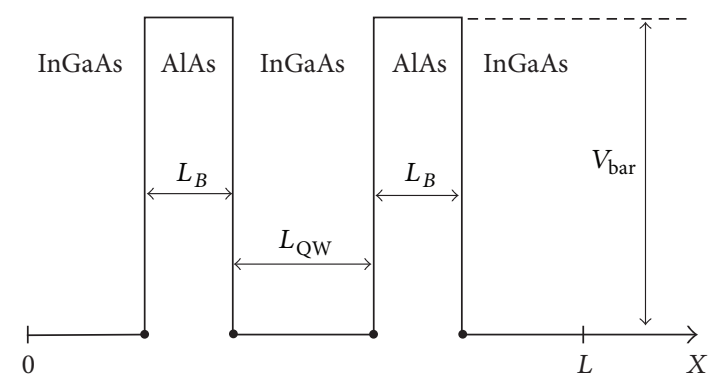

Figure 1: Potential of a double-barrier heterostructure.

\section{The Model}

We consider a one-dimensional two-barrier nanostructure schematically depicted in Figure 1. From the left of the emitter and from the right of the collector, the structure is bombarded by a flow of electrons. The RTD is exposed to the direct current $V(x)=-V_{\mathrm{dc}}(x / L)$ and alternating current $V_{t}(x, t)=$ $-V_{\mathrm{ac}} \cdot(x / L) \cos (\omega t)$ electric fields. The electron wave function matches the time-dependent Schrödinger equation:

$$
\begin{aligned}
\mathrm{i} \hbar \frac{\partial \psi_{k}(x, t)}{\partial t}= & -\frac{\hbar^{2}}{2 m^{*}} \frac{\partial \psi_{k}(x, t)}{\partial x} \\
& +\left(V_{\text {str }}(x)+V_{t}(x, t)+V(x)\right) \psi_{k}(x, t)
\end{aligned}
$$

The open boundary conditions for the time-dependent Schrödinger equation are used [17-19]. The current through the RTD is determined according to the following formula:

$$
\begin{gathered}
j(x, t)=\frac{e \hbar}{m^{*}} \int_{0}^{E_{\max }} g\left(E_{k}\right) \frac{\operatorname{Im}\left(\psi_{k}^{*}\left(\partial \psi_{k} / \partial x\right)\right)}{\sqrt{\left(2 m^{*} / \hbar^{2}\right) E_{k}}} d E_{k}, \\
g(k)=\frac{m^{*} k_{B} T}{2 \pi^{2} \hbar^{2}} \ln \left(1+\exp \left(\frac{E_{k}-E_{F}}{k_{B} T}\right)\right),
\end{gathered}
$$

where $g(k)$ is the Fermi function integrated over the transverse impulse, $m^{*}$ is the effective electron mass, $E_{F}$ is the Fermi energy, $T$ is the temperature, $E_{\max }=E_{F}+7 k_{B} T$, and $E_{k}=\hbar^{2} k^{2} / 2 m^{*}$.

After reaching the steady state when the current is periodically time-dependent, the expressions for an active component of the current $j_{c}$ with the same phase as the AC field and the reactive $j_{s}$ with the phase opposite to the phase of the AC field shall be determined as follows [11]:

$$
\begin{aligned}
& j_{c}(x)=\frac{\omega}{\pi} \int_{0}^{2 \pi / \omega} j(x, t) \cos (\omega t) d t \\
& j_{s}(x)=\frac{\omega}{\pi} \int_{0}^{2 \pi / \omega} j(x, t) \sin (\omega t) d t .
\end{aligned}
$$

We take into consideration the alternating currents' values averaged with respect to the coordinates $J_{c, s}=$ $(1 / L) \int_{0}^{L} j_{c, s}(x) d x$. The active current determines the real part of admittance $\left(\sigma=\operatorname{Re}(Y)=J_{c} / V_{\mathrm{ac}}\right)$ and the power delivered by the electrons to the field per period:

$$
P_{c}=\frac{\omega}{2 \pi} \int_{0}^{2 \pi / \omega} J(t) V_{\mathrm{ac}} \cos (\omega t) d t=\frac{V_{\mathrm{ac}} \cdot J_{c}}{2}=\frac{V_{\mathrm{ac}}^{2} \cdot \sigma}{2} .
$$


The reactive current determines the imaginary part of admittance $\left(\operatorname{Im}(Y)=J_{s} / V_{\mathrm{ac}}\right)$. This component is equivalently expressed as a reactance connected in parallel with $\sigma$. Here, we are interested in the negative values of $J_{c}$ corresponding to the electromagnetic field generation.

Also, we investigate the dependence of the active current $J_{c}{ }^{E}$ on the energy of the electrons. In this case, we write the expression for $j(x, t)$ substituting solely the electron contribution with the preset value of the energy; that is, $j(x, t)=$ $\operatorname{Im}\left(\psi_{k}^{*}\left(\partial \psi_{k} / \partial x\right)\right) / \sqrt{\left(2 m^{*} / \hbar^{2}\right) E_{k}} \cdot \ln \left(1+\exp \left(\left(E_{k}-E_{F}\right) / k_{B} T\right)\right)$ instead of the total current $j(x, t)$ integrated over the energy of the electrons. In the model with monoenergetic electrons we assume that $g\left(E_{k}\right)=\left(m^{*} / e \hbar\right) \cdot \delta\left(E_{k}-E\right)$. This leads to the following dimensionless expression for the current through RTD: $j(x, t)=\operatorname{Im}\left(\psi_{k}^{*}\left(\partial \psi_{k} / \partial x\right)\right) / \sqrt{\left(2 m^{*} / \hbar^{2}\right) E_{k}}$ and the values of $J_{0}, J_{c} \in[0,1]$.

The numerical calculations were carried out using the Crank-Nicolson method. In order to prevent the nonphysical reflections of the calculation area boundaries, we used the discrete transparent boundary conditions obtained for the Schrödinger difference equation [17-19]. The methods of numerical calculation are set out in detail in [17].

We consider the InGaAs/AlAs RTD structures described in [15]. These structures have an equal width of the quantum well $L_{\mathrm{QW}}=4.5 \mathrm{~nm}$ and equal heights of the barriers $V_{\mathrm{bar}}=$ $1.2 \mathrm{eV}$, but different widths of the quantum well's barriers. The thickness of the barriers for structure number 1 is $L_{b}=2 \mathrm{~nm}$. The thickness of the barriers for structure number 2 is $L_{b}=$ $1.4 \mathrm{~nm}$. The contacts' donor density is $n_{d}=1.3 \times 10^{18} \mathrm{~cm}^{-3}$ (Fermi energy is $E_{F}=0.1 \mathrm{eV}$ ).

\section{Results and Discussion}

3.1. The RTD Behaviour in a Weak AC Electric Field. Now, we shall consider the cases where the AC electric field is weak, that is, when the amplitude of its potential is $V_{\mathrm{ac}}=0.01 \Gamma \ll$ $\Gamma$. We shall compare the calculations in the model with the monoenergetic electrons and in the model with the Fermi distribution.

The results of the numerical calculations in the model with monoenergetic electrons showed that, at low frequencies, $\hbar \omega \ll \Gamma$, the active current is exactly described by the behaviour of the differential conductivity of the direct current $J_{c}=\left(V_{\mathrm{ac}} / 2\right)\left(\partial J_{0} / \partial E\right)$ (in this model, the role equivalent to the DC voltage is played by the energy of the electrons). Consequently, the maximum absolute value of the negative active current is reached in the maximum NDC. At high frequencies $\hbar \omega>\Gamma$, the active current is not described through the behaviour of the differential conductivity and reaches its maximum absolute value at the energy $\varepsilon \approx \varepsilon_{R}+$ $\hbar \omega$. This energy is beyond the area of the maximum NDC. Moreover, the value of the high-frequency active current in the area of the maximum NDC turns out to be less than the same in quantum regime (see Figure 2). These results are exactly consistent with the analytical theory [11] and are associated with the presence of quasi-energetic states that arise in an AC electric field and with the emitting transitions between these states.

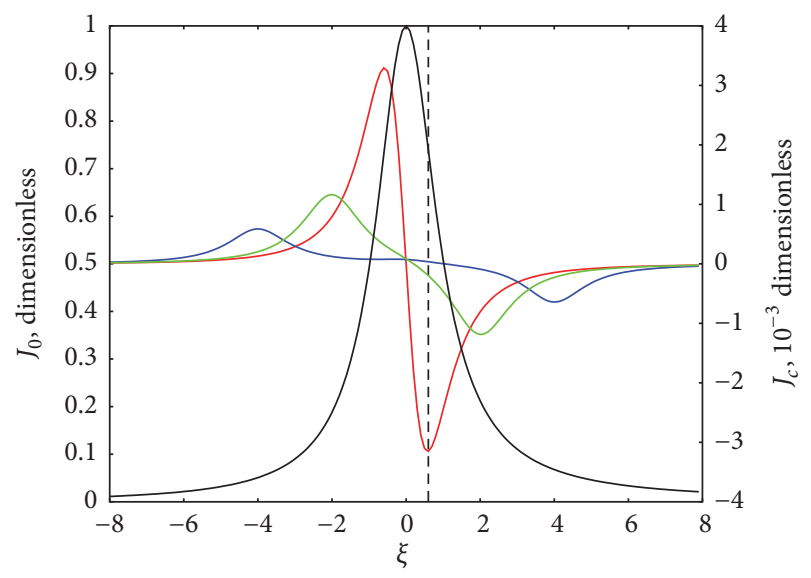

FIGURE 2: Direct current (solid black line, left $y$-axis) and active current $J_{c}$ (right $y$-axis) as a function of $\xi=\left(\varepsilon-\varepsilon_{R}\right) / \Gamma$ in the model with monoenergetic electrons at the following frequencies: $\hbar \omega=0.1 \Gamma$ (red line), $\hbar \omega=2 \Gamma$ (green line), and $\hbar \omega=4 \Gamma$ (blue line). Vertical dashed line indicates the area of maximum NDC.



FIgURE 3: Direct current (solid orange line, left $y$-axis) and active current $J_{c}$ (right $y$-axis) as a function of DC voltage for RTD heterostructure number 1 at the following frequencies: $\hbar \omega=4 \Gamma$ (black line), $\hbar \omega=8 \Gamma$ (red line), and $\hbar \omega=14 \Gamma$ (blue line). Vertical dashed line indicates the area of maximum NDC.

Now, we shall consider the model that takes into account the Fermi distribution of electrons over the energy states and the presence of the DC voltage. This model is more consistent with the experiment. At low frequencies $\hbar \omega \ll \Gamma$, the behaviour of the active current is still described by static differential conductivity, $J_{c}=V_{\mathrm{ac}}\left(\partial J_{0} / \partial V_{\mathrm{dc}}\right)$, and the position of the maximum absolute value of the negative active current $J_{c}$ (operating point position) coincides with the maximum NDC. Whereas, at high frequencies $\hbar \omega>\Gamma$, the Fermi distribution "smears" the regularities noted above, which is illustrated in Figure 3, the operating point position begins to depend on the relations $\varepsilon_{F} / \Gamma$ and $\omega / \Gamma$ and the temperature $T$.

Figures 4 and 5 depict the dependence of the operating point position relative to the maximum NDC which is designated by $\xi_{0}\left(\xi_{0}=0\right.$ corresponds to the maximum NDC), on the Fermi energy for different frequencies and 


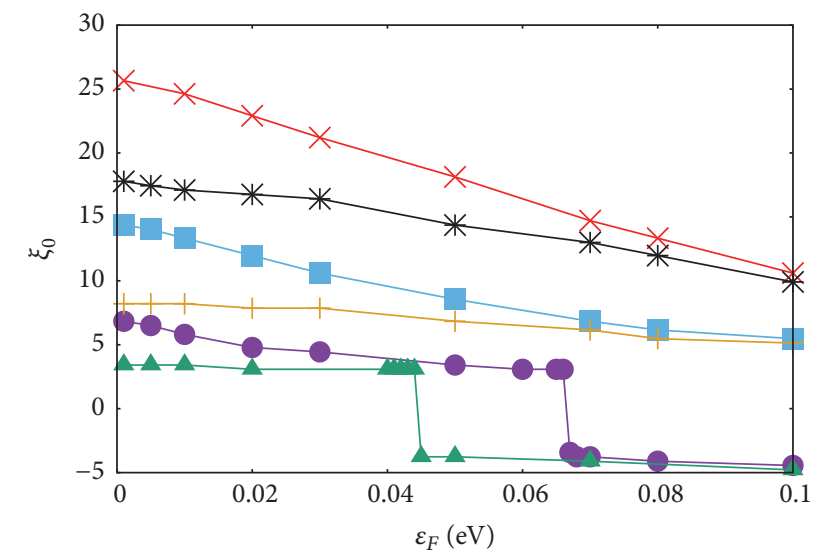

FIGURE 4: Operating point position relative to the maximum NDC as a function of the Fermi energy for RTD heterostructure number 1 at the following frequencies and temperatures: $\hbar \omega=4 \Gamma, T=300 \mathrm{~K}$ (green line); $\hbar \omega=4 \Gamma, T=0 \mathrm{~K}$ (purple line); $\hbar \omega=8 \Gamma, T=300 \mathrm{~K}$ (orange line); $\hbar \omega=8 \Gamma, T=0 \mathrm{~K}$ (blue line); $\hbar \omega=14 \Gamma, T=300 \mathrm{~K}$ (black line); $\hbar \omega=14 \Gamma, T=300 \mathrm{~K}$ (red line).



FIGURE 5: Operating point position relative to the maximum NDC as a function of the Fermi energy for RTD heterostructure number 2 at the following frequencies and temperatures: $\hbar \omega=2 \Gamma, T=300 \mathrm{~K}$ (orange line); $\hbar \omega=2 \Gamma, T=0 \mathrm{~K}$ (blue line); $\hbar \omega=4 \Gamma, T=300 \mathrm{~K}$ (black line); $\hbar \omega=4 \Gamma, T=0 \mathrm{~K}$ (red line).

temperatures $T=300 \mathrm{~K}, T=0 \mathrm{~K}$. We consider the structures number 1 and number 2 having different resonant energy level widths. The value of $\Gamma$ for the Structure number 2 is approximately 3.5 times higher than the corresponding value for the structure number 1 . In the case of structure number 1 (see Figure 4) that has the less value of $\Gamma$, for the AC field frequency $\hbar \omega=4 \Gamma$ it results that, at high values of the Fermi energy $\varepsilon_{F}=0.1 \mathrm{eV}$ (corresponds to the contacts' donor density $n_{d}=1.3 \times 10^{18} \mathrm{~cm}^{-3}$ ), the operating point is located to the left of the maximum NDC area (i.e., at a lower DC voltage). Moreover, the decrease of the Fermi energy does not significantly change the operating point position until the Fermi energy reaches a certain value $\varepsilon_{F}{ }^{(0)}$ that depends on the temperature. Upon reaching $\varepsilon_{F}{ }^{(0)}$, the operating point position will jump onto the area to the right of the maximum NDC. In the case of high frequencies $\hbar \omega=8 \Gamma, 16 \Gamma$, the

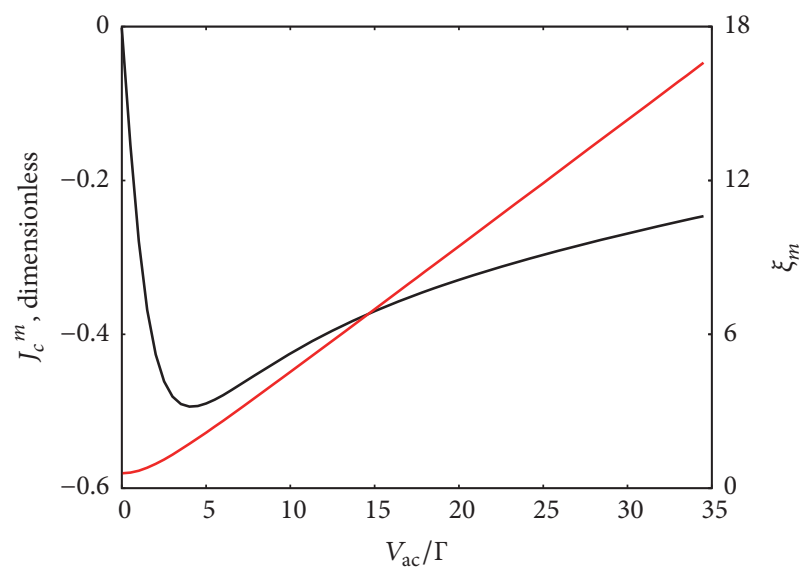

FIGURE 6: Maximum absolute value of the negative active current $J_{c}{ }^{m}$ (black line, left $y$-axis) and its position relative to the energy of the resonant level $\xi_{m}=\left(\varepsilon-\varepsilon_{R}\right) / \Gamma$ (red line, right $y$-axis) as a function of amplitude of the AC electric field in the model with monoenergetic electrons at the frequency $\hbar \omega=0.1 \Gamma$.

operating point position for all values of the Fermi energy under consideration is beyond the area of the maximum NDC to the right thereof. Reducing of $\varepsilon_{F}$ leads to the increase of $\xi_{0}$.

Moreover, this increase for $T=0$ is stronger than the corresponding value for $T=300 \mathrm{~K}$. The dependence of $\xi_{0}\left(\varepsilon_{F}\right)$ for structure number 2 , having a higher resonant energy level width, is shown in Figure 5. It can be seen that the operating point position already for $\hbar \omega=2 \Gamma$ is beyond the area of the maximum NDC.

Thus, when taking into account the Fermi distribution in the case of high frequencies $\hbar \omega>\Gamma$, the increase of the resonant energy level width (i.e., the decrease in the ratio $\varepsilon_{F} / \Gamma$ ) leads to the result corresponding to the one obtained within the framework of the relevant model with monoenergetic electrons. Also, the temperature does only have an effect for low values of the Fermi energy.

3.2. The RTD Behaviour in a Strong AC Electric Field. We shall investigate the RTD behaviour in a strong AC electric field when the amplitude of its potential is $V_{\mathrm{ac}}>\Gamma$. First, we shall study the basic features specific to the model of the monoenergetic electrons. We shall take into account the behaviour of the maximum absolute value of the negative active current that will be denoted as $J_{c}{ }^{m}$ and its position relative to the energy of the resonant level $\xi_{m}$ depending on the amplitude of the AC electric field $V_{\text {ac }}$.

At low frequencies $\hbar \omega \ll \Gamma$ (see Figure 6), the absolute value of $J_{c}{ }^{m}$ increases as the AC field amplitude increases and reaches its maximum value at $V_{\mathrm{ac}} \approx 4 \Gamma$. The further increase of $V_{\mathrm{ac}}$ reduces $J_{c}{ }^{m}$. Moreover, the operating point position $\xi_{m}$ monotonically tends toward higher values as the field amplitude increases.

In the case of high frequencies, as opposed to [14], we shall not fix the operating point position $\xi=\hbar \omega$. Instead, the operating point position will be determined in accordance with the position of the maximum absolute value of the negative 


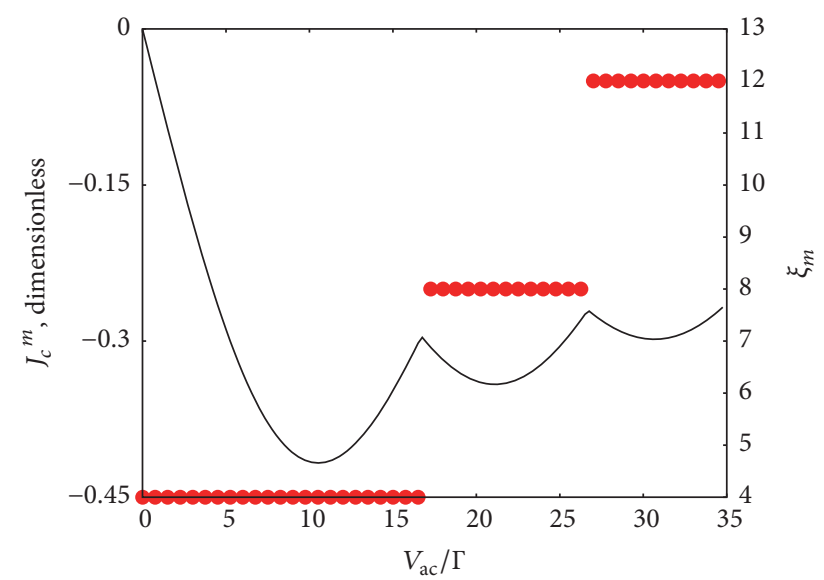

FIGURE 7: Maximum absolute value of the negative active current $J_{c}{ }^{m}$ (solid line, left $y$-axis) and its position relative to the energy of the resonant level $\xi_{m}=\left(\varepsilon-\varepsilon_{R}\right) / \Gamma$ (red circles, right $y$-axis) as a function of amplitude of the AC electric field in the model with monoenergetic electrons at the frequency $\hbar \omega=4 \Gamma$.

active current $J_{c}$. The numerical calculations are summarised in Figure 7 for frequency $\hbar \omega=4 \Gamma$. The dependence of the absolute value of current $J_{c}{ }^{m}$ on the AC electric field amplitude $V_{\mathrm{ac}}$ is characterised with a series of maximums with the amplitude decreasing as the field increases, wherein the operating point position $\xi_{m}$ at the transition from one maximum to another maximum abruptly changes by the value $\hbar \omega$. Thus, at an increase in the alternating current amplitude, the larger contribution to the active current $J_{c}$ is being provided by the electrons with energies $\varepsilon=\varepsilon_{R}+n \hbar \omega$, $n=2,3, \ldots$; that is, there is an excitement of the higher quasienergetic levels. Consequently, in strong AC electric fields the best regime is the quantum regime associated with the presence of transitions between the states with quasi-energies $\varepsilon_{R}+n \hbar \omega$. It is worth noting that the maximum absolute value of the negative active current is $\approx 0.42$ of the direct current in resonance, which allows reliance on achieving high output powers at high frequencies.

Now, we shall consider the case of the Fermi distribution of electrons over the energy states in the presence of the DC voltage. In this case, as above, we shall denote the maximum absolute value of the negative active current $J_{c}$, through $J_{c}{ }^{m}$, and shall denote the corresponding DC voltage as $V_{\mathrm{dc}} m$.

First, we investigate RTD nanostructure number 2 having the highest resonant energy level width of all the structures under our consideration. Figure 8 shows the dependence of $J_{c}{ }^{m}$ and $V_{\mathrm{dc}}{ }^{m}$ on the field amplitude $V_{\mathrm{ac}}$ for the frequencies $\hbar \omega=0.1 \Gamma$ and $\hbar \omega=2 \Gamma$. Taking into consideration the Fermi distribution of electrons over the energy states leads to the fact that the character of the dependence $J_{c}{ }^{m}$ on the field amplitude is qualitatively similar to these frequencies. Moreover, at high amplitudes of the AC electric field, the operating point position behaves in the same way for both frequencies. Within the entire range of the considered field amplitudes, $J_{c}{ }^{m}$ is lower for the higher frequency but its droop with the increasing field amplitude is also lower. This is because, at $\hbar \omega=2 \Gamma$, the quantum regime is implemented.

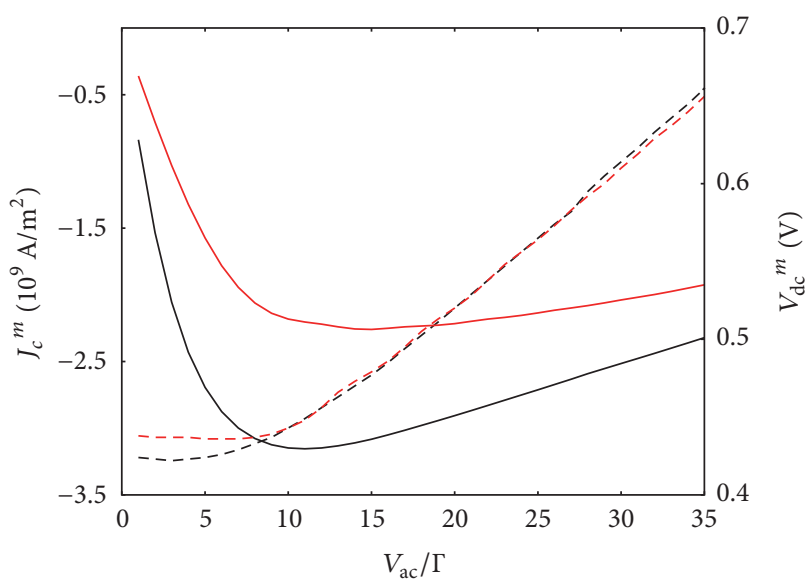

FIGURE 8: Maximum absolute value of the negative active current $J_{c}^{m}$ (solid line, left $y$ axis) and its position relative to the energy of the resonant level $\xi_{m}=\left(\varepsilon-\varepsilon_{R}\right) / \Gamma$ (dashed line, right $y$ axis) as a function of amplitude of the AC electric field for RTD heterostructure number 2 at the following frequencies: $\hbar \omega=0.1 \Gamma$ (black line) and $\hbar \omega=2 \Gamma$ (red line).

The features of this quantum regime are "smeared" by the Fermi distribution. At high field amplitudes, the contribution into the active current is being provided by the higher quasienergetic levels. This effect is much stronger for structure number 1 with a lower resonant energy level width (see Figure 9(a)). Figure 9(b) shows the dependence of active current $J_{c}{ }^{E}$ on the energy of the electrons corresponding to the amplitude of the field $V_{\mathrm{ac}}=25 \Gamma$ for RTD heterostructure number 1 at the frequency $\hbar \omega=4 \Gamma$. This dependence makes it clear that there are several maxima of $J_{c}{ }^{E}$ absolute value distanced from each other by $\hbar \omega$.

In investigating RTD structure number 2, the increase in the $\mathrm{AC}$ electric field frequency to $\hbar \omega=4 \Gamma$ leads to a qualitative change of the dependence $J_{c}{ }^{m}$ on $V_{\text {ac }}$ (see Figure 10) compared with $\hbar \omega=0.1 \Gamma, 2 \Gamma$. Now, similarly to the case of the monoenergetic electrons, there is a series of $J_{c}{ }^{m}$ absolute value maxima with the amplitude decreasing with the increasing field and the operating point position at the transition from one maximum to another maximum abruptly changes by $\approx 4 \Gamma$ for the first maximum and by $\approx 3 \Gamma$ for the second maximum, that is, by the value close to the AC field's frequency $\hbar \omega=4 \Gamma$.

An important result is that, within a wide frequency range, the active current reaches a high value comparable with the maximum direct current in the absence of the AC electric field $\left(j_{0 \max } \approx 6 \cdot 10^{9} \mathrm{~A} / \mathrm{m}^{2}\right)$. The active current can reach high values in a strong field for high frequencies that indicates the implementation of the quantum regime of the RTD. The second important result is the stability of the maximum $J_{c}$ to the strong electromagnetic fields; that is, $J_{c}{ }^{m}$ drops slightly as the field amplitude increases.

\section{Conclusion}

In the presented study, we analyse the behaviour of InGaAs/AlAs double-barrier nanostructure in AC electric 


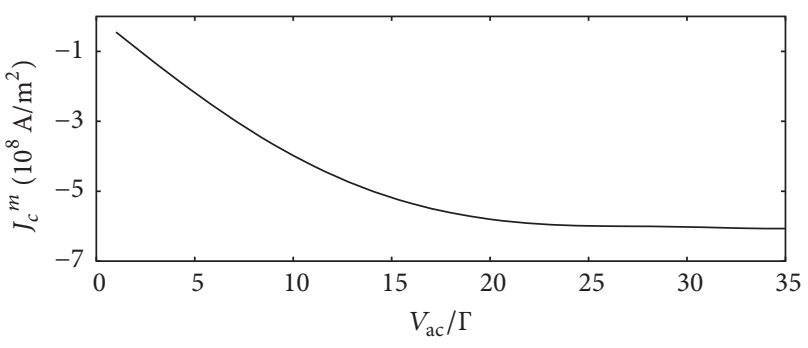

(a)

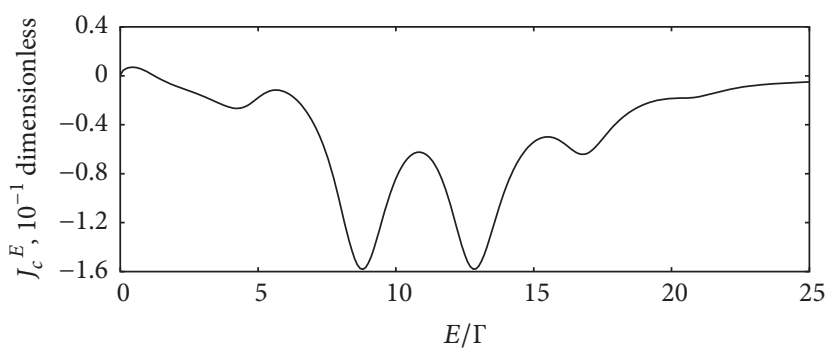

(b)

Figure 9: (a) Maximum absolute value of the negative active current current $J_{c}{ }^{m}$ as a function of amplitude of the AC electric field for RTD heterostructure number 1 at the frequency $\hbar \omega=4 \Gamma$. (b) Dimensionless electron contribution to the active current as a function of the energy of the electron for amplitude of the AC electric field $V_{\mathrm{ac}}=25 \Gamma$, frequency $\hbar \omega=4 \Gamma$, and RTD heterostructure number 1 .

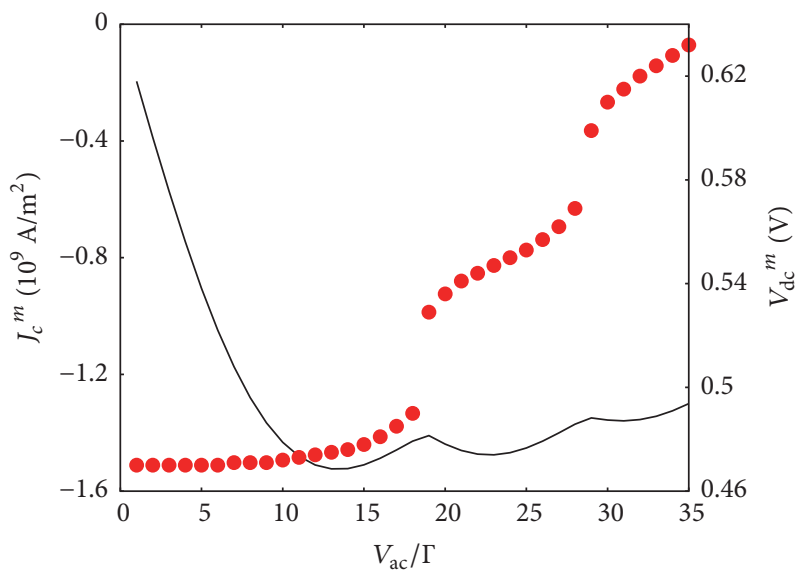

FIGURE 10: Maximum absolute value of the negative active current $J_{c}^{m}$ (solid line, left $y$ axis) and operating point position (red circles, right $y$ axis) as a function of amplitude of the AC electric field at the frequency $\hbar \omega=4 \Gamma$ for RTD heterostructure number 2 .

field in wide range of frequencies and field amplitudes using coherent quantum-mechanical model with open boundary conditions.

Within the low-frequency limit $\hbar \omega \ll \Gamma$ for weak AC electric fields, the current's active component of the RTD shall be determined by the differential conductivity. In strong fields when the field potential amplitude is higher than the resonant energy level width $V_{\mathrm{ac}}>\Gamma$, the active current reaches its maximum absolute value at a certain value of the field amplitude which depends on the ratio $\varepsilon_{F} / \Gamma$, that is, on the parameters of the active region and on the contacts' donor density. Moreover, the value of the DC voltage that results in the maximum absolute value of the active current monotonically increases as the field amplitude increases.

In the case of high frequencies $\hbar \omega>\Gamma$, it is shown that the maximum absolute value of the active current is associated with the implementation of the quantum regime. At the same time, ratio $\varepsilon_{F} / \Gamma$ also has a significant impact on the behaviour of the active current $J_{c}$. The structures with a lower value of this ratio at lower frequencies begin to present features typical for monoenergetic distribution. At a small signal approximation $V_{\text {ac }} \ll \Gamma$, these features are associated with the displaced operating point position from the area of the maximum NDC towards the higher values of the DC voltage. In strong AC electric fields, the RTD active current at high frequencies can reach significant values comparable to the maximum direct current. Moreover, at high amplitudes of the field the impact to the active current gives the higher quasi-energetic levels that results in a slow droop of the active current as the field amplitude increases. In addition, this leads to the abrupt changes of the operating point position by the value $\hbar \omega$. This ensures the achievement of relatively high powers of the RTD generation order $10^{5} \mathrm{~W} / \mathrm{cm}^{2}$ at high frequencies.

\section{Conflicts of Interest}

The authors declare that they have no conflicts of interest.

\section{Acknowledgments}

The reported study was funded by RFBR according to the Research Project no. 16-32-00588 mol_a.

\section{References}

[1] L. L. Chang, L. Esaki, and R. Tsu, "Resonant tunneling in semiconductor double barriers," Applied Physics Letters, vol. 24, p. $593,1974$.

[2] M. Tsuchiya, H. Sakaki, and J. Yoshino, "Room temperature observation of differential negative resistance in an alas/gaas/alas resonant tunneling diode," Japanese Journal of Applied Physics, vol. 24, no. 6 A, pp. L466-L468, 1985.

[3] T. C. Sollner, P. E. Tannenwald, D. D. Peck, and W. D. Goodhue, "Quantum well oscillators," Applied Physics Letters, vol. 45, no. 12, pp. 1319-1321, 1984.

[4] H. Kanaya, H. Shibayama, R. Sogabe, S. Suzuki, and M. Asada, "Fundamental oscillation up to $1.31 \mathrm{THz}$ in resonant tunneling diodes with thin well and barriers," Applied Physics Express, vol. 5, no. 12, Article ID 124101, 2012.

[5] H. Kanaya, R. Sogabe, T. Maekawa, S. Suzuki, and M. Asada, "Fundamental oscillation up to $1.42 \mathrm{Thz}$ in resonant tunneling diodes by optimized collector spacer thickness," Journal of Infrared, Millimeter, and Terahertz Waves, vol. 35, no. 5, pp. 425431, 2014. 
[6] T. Maekawa, H. Kanaya, S. Suzuki, and M. Asada, "Frequency increase in terahertz oscillation of resonant tunnelling diode up to $1.55 \mathrm{THz}$ by reduced slot-antenna length," Electronics Letters, vol. 50, no. 17, pp. 1214-1216, 2014.

[7] T. Maekawa, H. Kanaya, S. Suzuki, and M. Asada, "Oscillation up to $1.92 \mathrm{THz}$ in resonant tunneling diode by reduced conduction loss," Applied Physics Express, vol. 9, no. 2, Article ID 024101, 2016.

[8] R. K. Mains and G. I. Haddad, "Time-dependent modeling of resonant-tunneling diodes from direct solution of the Schrödinger equation," Journal of Applied Physics, vol. 64, no. 7, pp. 3564-3569, 1988.

[9] R. K. Mains and G. I. Haddad, "Wigner function modeling of resonant tunneling diodes with high peak-to-valley ratios," Journal of Applied Physics, vol. 64, no. 10, pp. 5041-5044, 1988.

[10] D. Sokolovski, "Resonance tunneling in a periodic timedependent external field," Physical Review B, vol. 37, no. 8, pp. 4201-4208, 1988.

[11] V. F. Elesin, "Theory of coherent generation in resonanttunneling diodes," Journal of Experimental and Theoretical Physics, vol. 89, no. 2, pp. 377-383, 1999.

[12] Y. B. Zel'dovich, "The quasienergy of a quantum-mechanical system subjected to a periodic action," Journal of Experimental and Theoretical Physics, vol. 51, article 1492, 1967.

[13] V. F. Elesin, I. Y. Kateev, A. V. Krasheninnikov, and A. I. Podlivaev, "Theory of coherent oscillations in a resonant tunneling diode," Physics-Uspekhi, vol. 43, no. 3, pp. 291-293, 2000.

[14] V. F. Elesin, "Resonant tunneling and a nonlinear response in RF fields," Journal of Experimental and Theoretical Physics, vol. 97, no. 2, pp. 343-357, 2003.

[15] V. V. Kapaev, Y. V. Kopaev, S. A. Savinov, and V. N. Murzin, "High-frequency response and the possibilities of frequencytunable narrow-band terahertz amplification in resonant tunneling nanostructures," Journal of Experimental and Theoretical Physics, vol. 116, no. 3, pp. 497-515, 2013.

[16] V. V. Kapaev, "Nonlinear theory of the narrow-band generation and detection of terahertz radiation in resonant tunneling heterostructures," Journal of Experimental and Theoretical Physics, vol. 121, no. 2, pp. 303-320, 2015.

[17] J.-F. Mennemann, A. Jungel, and H. Kosina, "Transient Schrodinger-Poisson simulations of a high-frequency resonant tunneling diode oscillator," Journal of Computational Physics, vol. 239, pp. 187-205, 2013.

[18] A. Arnold, "Mathematical concepts of open quantum boundary conditions," Transport Theory and Statistical Physics, vol. 30, no. 4-6, pp. 561-584, 2001.

[19] A. Arnold, M. Ehrhardt, and I. Sofronov, "Discrete transparent boundary conditions for the Schrodinger equation: fast calculation, approximation, and stability," Communications in Mathematical Sciences, vol. 1, no. 3, pp. 501-556, 2003. 

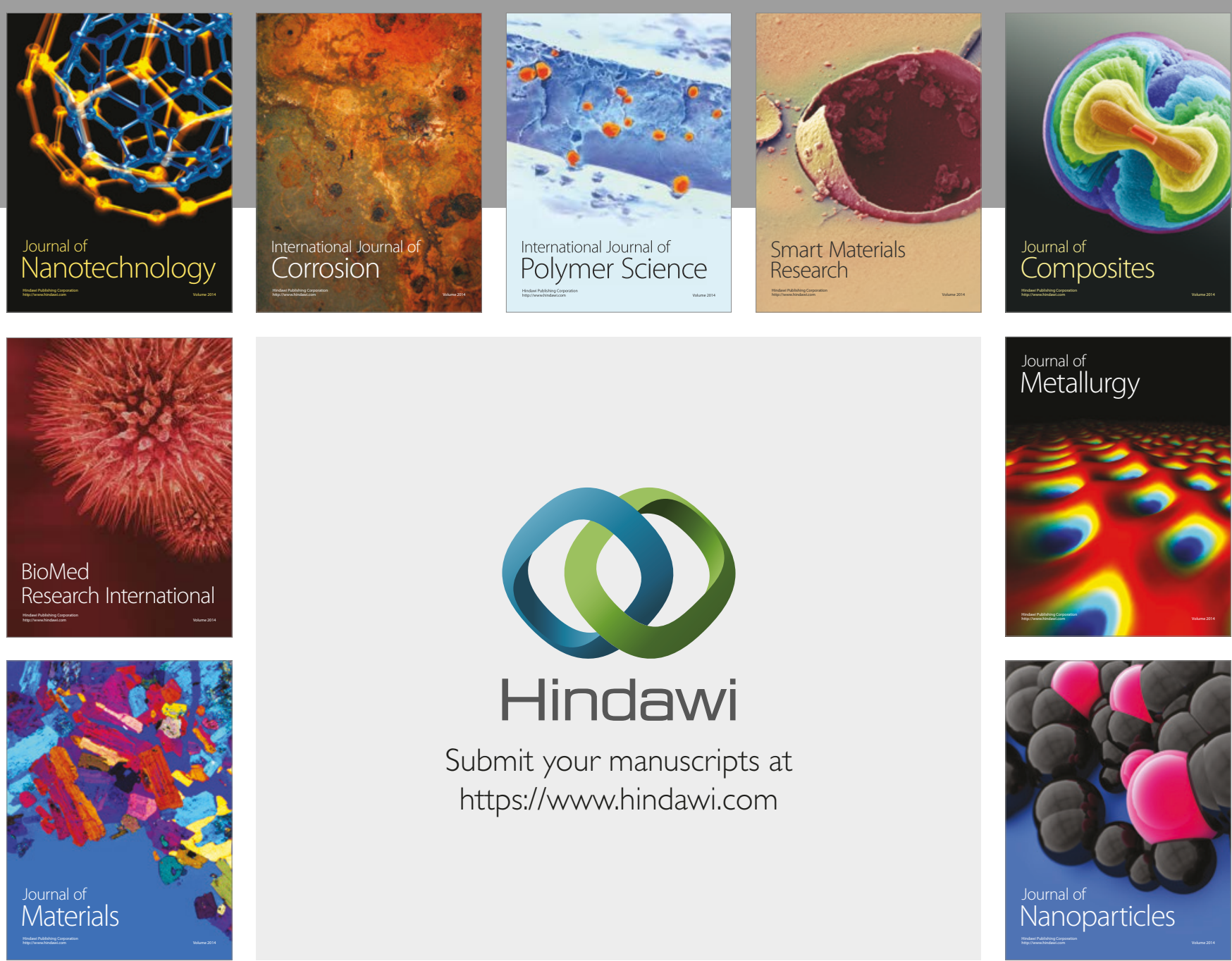

\section{Hindawi}

Submit your manuscripts at

https://www.hindawi.com
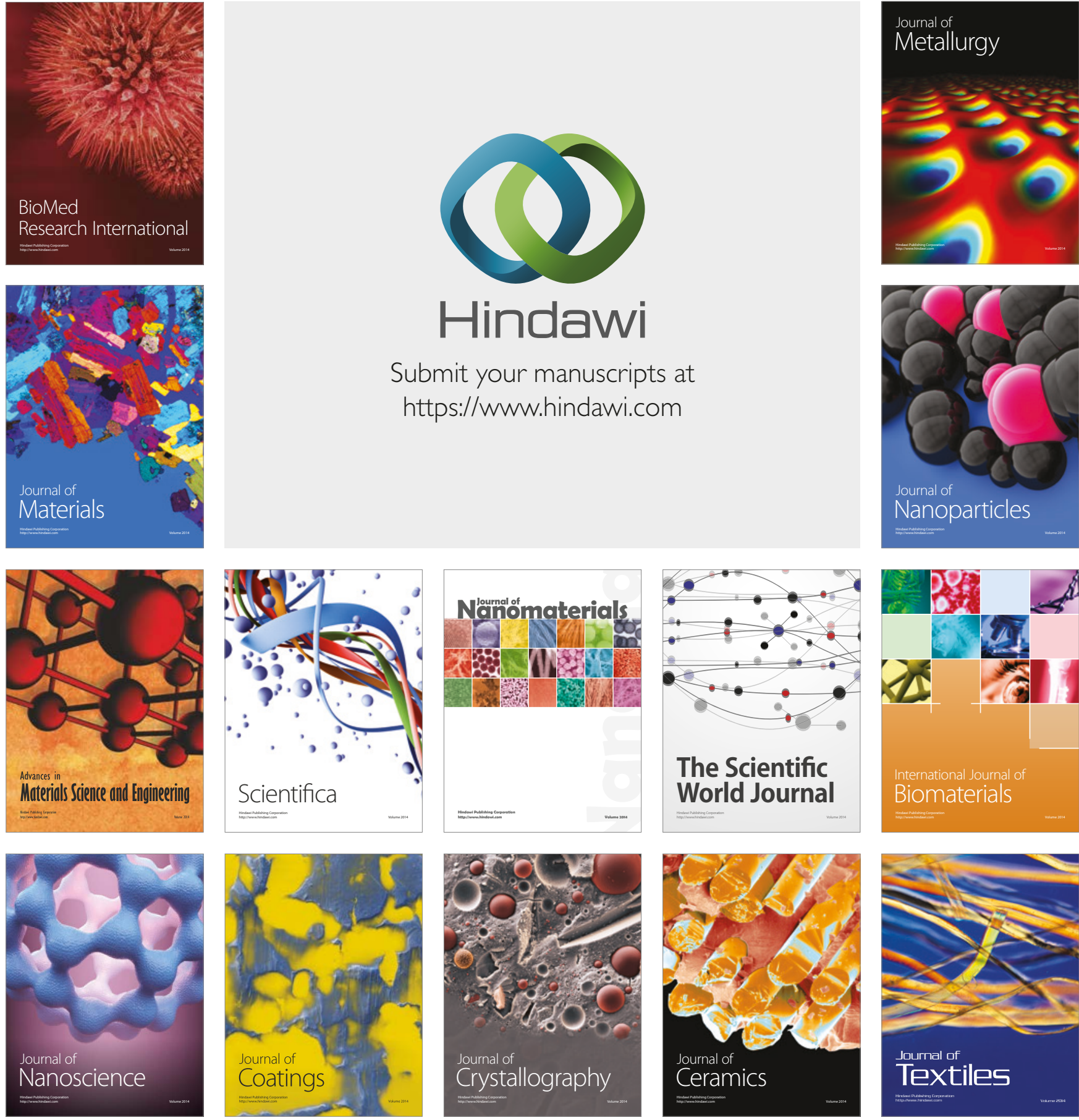



The Scientific World Journal
